doi: $10.13108 / 2021-13-2-152$

\title{
YAMILOV'S THEOREM FOR DIFFERENTIAL AND DIFFERENCE EQUATIONS
}

\author{
DECIO LEVI, MIGUEL A. RODRÍGUEZ
}

\begin{abstract}
S-integrable scalar evolutionary differential difference equations in $1+1$ dimensions have a very particular form described by Yamilov's theorem. We look for similar results in the case of S-integrable 2-dimensional partial difference equations and 2-dimensional partial differential equations. To do so, on one side we discuss the semi-continuous limit of S-integrable quad equations and on the other, we semi-discretize partial differential equations. For partial differential equations, we show that any equation can be semi-discretized in such a way to satisfy Yamilov's theorem. In the case of partial difference equations, we are not able to find a form of the equation such that its semi-continuous limit always satisfies Yamilov's theorem. So we just present a few examples, in which to get evolutionary equations, we need to carry out a skew limit. We also consider an S-integrable quad equation with non-constant coefficients which in the skew limit satisfies an extended Yamilov's theorem as it has non-constant coefficients. This equation turns out to be a subcase of the Yamilov discretization of the Krichever-Novikov equation with non-constant coefficient, an equation suggested to be integrable by Levi and Yamilov in 1997 and whose integrability has been proved only recently by algebraic entropy. If we do a strait limit, we get non-local evolutionary equations, which show that an extension of Yamilov's theorem may exist in this case.
\end{abstract}

Keywords: differential difference equations, continuous and discrete integrable systems, Yamilov's theorem.

Mathematics Subject Classification: 39A14, 35Q53

\section{INTRODUCTION}

Integrability properties of differential difference (D $\Delta \mathrm{Es})$ or partial difference equations (P $\Delta \mathrm{Es}$ ) have been thoroughly studied along the last decades, following several approaches and criteria to characterize these equations (see, among many others, references [1], 6], [9], [13]). In the case of $1+1 \mathrm{D} \Delta \mathrm{Es}$, Yamilov's theorem [10], [11], [13] provides a necessary condition for the existence of a large number of conserved quantities of S-integrable equations [5]. This is reflected in a certain symmetry of the points involved in the equation.

By this work, we start a project aiming on characterizing S-integrable equations in total, i.e. non only D $\Delta$ Es but also either partial differential equations (PDEs) or P $\Delta$ Es.

D. Levi, M.A. Rodriguez, Yamilov's theorem for differential and difference equations.

(C) D. Levi, M.A. Rodriguez. 2021.

MAR was partially supported by Spain's Ministerio de Ciencia, Innovación y Universidades under grant PGC2018-094898-B-I00, as well as by Universidad Complutense de Madrid under grant G/6400100/3000.

Submitted March 11, 2021. 
As a part of this project, we plan to extend Yamilov's result to other classes of equations. To do so, in this work we provide some examples of integrable quad equations whose partial continuous limits satisfy Yamilov's condition. Moreover, we show that each PDE can be partially discretized into a D $\Delta \mathrm{E}$, which satisfies Yamilov's theorem.

In Section 2 we present a brief description of Yamilov's theorem and in the following Section its consequences for PDEs. Section 4 is devoted to the partial continuous limit for quad-graph equations, P $\Delta$ Es defined on a square, and in Section 5 we summarize our results and present some plans for future works on this project.

\section{YAMILOV'S THEOREM}

As we have written above, Yamilov's theorem gives a necessary condition for a $1+1 \mathrm{D} \Delta \mathrm{Es}$ to admit higher order conservation laws. Following [10], [11], [13], the search for these equations yields the conclusion that only symmetrical (in a sense to be detailed below) equations have this property. Let us consider a one-dimensional lattice, labeled by $n \in \mathbb{Z}$, and a function $u_{n}(t)$ of a continuous variable $t$ (real or complex), which takes values at the lattice points. We are interested in $\mathrm{D} \Delta \mathrm{Es}$ of the type:

$$
\dot{u}_{n}=f\left(u_{n+N}, u_{n+N-1}, \ldots, u_{n+M}\right) \equiv f_{n},
$$

where the dot above $u_{n}$ denotes the derivative with respect to $t, N>M$ and the function $f$ truly depends on $u_{n+N}$ and $u_{n+M}$. A conservation law for this equation is a relation of the form:

$$
D_{t} p_{n}=(T-1) q_{n},
$$

where $p_{n}$, the conserved density, and $q_{n}$ are functions of $\left(u_{n}, u_{n+1}, \ldots, u_{n+k}\right)(k$ is a finite integer number called the order),

$T$ is the shift operator, $\left(T \phi_{n}=\phi_{n+1}\right)$ and $D_{t}$ the total derivative with respect to $t$ :

$$
D_{t}=\sum_{i=0}^{k} f_{n+i} \frac{\partial}{\partial u_{n+i}} .
$$

Yamilov's theorem provides a condition on the form of equation 2.1 ensuring that it has conservation laws.

Theorem 2.1. If (2.1) possesses a conservation law of order $m>\min \{|N|,|M|\}$, then $N=-M, N>0$.

The proof of this theorem is based on a detailed studying of the variational derivative of the conserved density $p_{n}$ of equation (2.1), which exists due to its S-integrability, see [10], [11], [13].

\section{Discussion of PDEs from the Point of VIEW OF TheOREM 2.1}

According Theorem 2.1, if 2.1) is an S-integrable D $\Delta \mathrm{E}$ and if the function $f_{n}$ contains the highest shift $u_{n+N}$, then it should also contain a lowest shift $u_{n-N}$. Defining

$$
v_{k}^{( \pm)}=u_{n+k} \pm u_{n-k},
$$

we can rewrite (2.1) in the S-integrable case as

$$
\dot{u}_{n}=f\left(v_{N}^{(+)}, v_{N}^{(-)}, v_{N-1}^{(+)}, v_{N-1}^{(-)}, \cdots, v_{1}^{(+)}, v_{1}^{(-)}, u_{n}\right) .
$$

To perform the continuous limit, we define $x=n h$ and, as $h \rightarrow 0$, we write the following Taylor expansion

$$
u_{n \pm j}=w(x \pm j h)=w(x) \pm j h w_{x}+\frac{1}{2} j^{2} h^{2} w_{2 x} \pm \frac{1}{3 !} j^{3} h^{3} w_{3 x}+\frac{1}{4 !} j^{4} h^{4} w_{4 x}+\cdots,
$$


where $w_{n x}$ is the $n$-th derivative of $w(x)$ with respect to $x$. In this way, we can write a list of Taylor expansions for $v_{j}^{( \pm)}$. For the lowest values of $j$ we have:

$$
\begin{aligned}
& v_{1}^{(+)}=2 w(x)+h^{2} w_{2 x}+\frac{h^{4}}{12} w_{4 x}+\cdots, \\
& v_{1}^{(-)}=2 h w_{x}+\frac{1}{3} h^{3} w_{3 x}+\cdots, \\
& v_{2}^{(+)}=2 w(x)+4 h^{2} w_{2 x}+\frac{4}{3} h^{4} w_{4 x}+\cdots, \\
& v_{2}^{(-)}=4 h w_{x}+\frac{8}{3} h^{3} w_{3 x}+\cdots, \\
& v_{3}^{(+)}=2 w(x)+9 h^{2} w_{2 x}+\frac{27}{4} h^{4} w_{4 x}+\cdots
\end{aligned}
$$

Consequently, we can express all derivatives of the function $w(x)$ in terms of the Taylor expansions of $v_{j}^{( \pm)}$up to order $h^{2}$. Here we present the leading terms:

$$
\begin{aligned}
w_{x} & =\frac{1}{2 h} v_{1}^{(-)}+\mathcal{O}\left(h^{2}\right), \\
w_{2 x} & =\frac{1}{3 h^{2}}\left[v_{2}^{(+)}-v_{1}^{(+)}\right]+\mathcal{O}\left(h^{2}\right), \\
w_{3 x} & =\frac{1}{2 h^{3}}\left[v_{2}^{(-)}-2 v_{1}^{(-)}\right]+\mathcal{O}\left(h^{2}\right), \\
w_{4 x} & =\frac{1}{10 h^{4}}\left[3 v_{3}^{(+)}-8 v_{2}^{(+)}+5 v_{1}^{(+)}\right]+\mathcal{O}\left(h^{2}\right) .
\end{aligned}
$$

As all continuous derivatives can be expressed in terms of symmetric differences, by Theorem 2.1, we do have no constraints on the form of an S-integrable PDE. This result agrees with what we know about S-integrable PDEs.

\section{Discussion of P $\Delta$ Es from the point of View of Theorem 2.1}

In this section we discuss by examples the semi-continuous limit of S-integrable P $\Delta$ Es.

4.1. Lattice potential Korteweg-de Vries equation. As an introduction to the procedure necessary to do the semi-continuous limit, we consider the well known example of the lattice potential Korteweg-de Vries $(\mathrm{lpKdV})$ equation [2], [6], [12]:

$$
\left(u_{n, m+1}-u_{n+1, m}\right)\left(u_{n, m}-u_{n+1, m+1}\right)=p^{2}-q^{2},
$$

where $u_{n, m}$ are the values of the variable $u$ at the points of a two dimensional lattice labeled by the integers $n, m \in \mathbb{Z}$. Equation (4.1) is nothing else but the nonlinear superposition formula for the KdV. The parameters $p, q$ are obtained in the construction of the lpKdV as the parameters of the sequence of two Bäcklund transformations which give the nonlinear superposition formula. The lpKdV satisfies the integrability property provided by the compatibility around the cube as shown in [2].

On (4.1) we will carry out a procedure to transform the discrete index $m$ into a continuous variable $t$ in such a way that equation (4.1) becomes an evolutionary $\mathrm{D} \Delta \mathrm{E}$ for the unknown function $u_{k}(t)$ depending on a continuous variable $t$ and a discrete index $k$.

The base of the method is the Taylor expansion in a parameter $\epsilon$ of $u_{n, m}$ at a particular solution $u_{0}$ of the equation. Both the index $m$ and the corresponding parameter $q$ will depend on $\epsilon$. As a particular solution, it is convenient to use a simple function which, in this case, is given by $u_{0}=p n+q m$. By the change of variables

$$
u_{n, m}=v_{n, m}-u_{0}
$$


equation (4.1) becomes

$$
\left(p-q+v_{n, m+1}-v_{n+1, m}\right)\left(p+q+v_{n, m}-v_{n+1, m+1}\right)=p^{2}-q^{2},
$$

which has as a solution $v_{0}=0$.

A simple approach to the continuous limit when $m$ goes to infinity and $\epsilon$ goes to zero in such a way that $m \epsilon$ is finite is the following. We define a new function $V_{n}(t)=v_{n, m}$ and a continuous variable $t=t_{0}+m \epsilon$ such that

$$
v_{n, m+j}=V_{n}(t)+j \epsilon \dot{V}_{n}(t)+O\left(\epsilon^{2}\right) .
$$

Moreover, we define a parameter $q$ as

$$
q=\epsilon^{-1} .
$$

Substituting (4.4), (4.5) into (4.3), we see that coefficient at $\epsilon^{-1}$ vanishes, while the zero order term yields the equation

$$
\dot{V}_{n}+\dot{V}_{n+1}=\left(V_{n+1}-V_{n}\right)\left[2 p-\left(V_{n+1}-V_{n}\right)\right] .
$$

Equation (4.6) is a nonlocal $\mathrm{D} \Delta \mathrm{E}$ which has derivatives with respect to $t$ at two different points of the lattice $n$ and $n+1$. To obtain an evolutionary $\mathrm{D} \Delta \mathrm{E}$ with a derivative just at one point, we have to mix the indices in the lattice, a skew limit as is called in [6]. We let $k=n+m$ and introduce a new variable $w_{k, m}$ such that

$$
v_{n+i, m+j}=w_{k+i+j, m+j} .
$$

Then 4.3 is transformed into

$$
\left(p-q+w_{k+1, m+1}-w_{k+1, m}\right)\left(p+q+w_{k, m}-w_{k+2, m+1}\right)=p^{2}-q^{2} .
$$

In this case, defining as above $U_{k}(t)=w_{k, m}$ with $q=p+\epsilon$, the $\epsilon^{0}$ term vanishes and at first order we get:

$$
\dot{U}_{k}=\frac{U_{k-1}-U_{k+1}}{U_{k-1}-U_{k+1}+2 p} .
$$

Equation $(4.9)$ is an evolutionary $\mathrm{D} \Delta \mathrm{E}$, with terms at points $k-1, k$ and $k+1$, which thus satisfies Yamilov's condition of S-integrability.

4.2. Integrable map obtained by factorization. Let us consider a nonlinear $\mathrm{P} \Delta \mathrm{E}$ :

$$
\begin{aligned}
c_{1} u_{n, m} u_{n+1, m} & +c_{2}\left(u_{n, m} u_{n+1, m+1}+u_{n+1, m} u_{n, m+1}\right)+c_{3} u_{n, m+1} u_{n+1, m+1} \\
& +c_{5} u_{n, m} u_{n, m+1}+c_{6} u_{n+1, m} u_{n+1, m+1}=0,
\end{aligned}
$$

an example taken from [7], [1]. Equation (4.10) has been proven to be integrable for all values of constants $c_{i}$ by checking its algebraic entropy.

In the particular case $c_{1}=c_{5}$ and $c_{6}=c_{3}$, the equation is invariant under the swapping of $n$ and $m$.

4.2.1. Continuous limit $t=\epsilon m$ and $u_{n, m}=v_{n}(t)$. We have:

$$
\begin{aligned}
c_{5} v_{n}^{2} & +c_{6} v_{n+1}^{2}+\left(c_{1}+2 c_{2}+c_{3}\right) v_{n} v_{n+1} \\
& +\epsilon\left(c_{5} v_{n} \dot{v}_{n}+c_{6} v_{n+1} \dot{v}_{n+1}+\left(c_{2}+c_{3}\right)\left(v_{n+1} \dot{v}_{n}+v_{n} \dot{v}_{n+1}\right)\right)+\mathcal{O}\left(\epsilon^{2}\right)=0 .
\end{aligned}
$$

The terms of zero order in $\epsilon$ do not involve the derivatives in $t$. Hence, in order to get a $\mathrm{D} \Delta \mathrm{E}$, we have to suppose that the coefficients $c_{i}$ depend on $\epsilon$. We have the following possibilities:

1. $c_{5}=\alpha_{5} \epsilon, c_{6}=\alpha_{6} \epsilon, c_{1}+2 c_{2}+c_{3}=0$,

$$
\left(c_{1}+c_{2}\right) \frac{\mathrm{d}}{\mathrm{d} t}\left(v_{n} v_{n+1}\right)=\alpha_{5} v_{n}^{2}+\alpha_{6} v_{n+1}^{2}=0,
$$


2. $c_{5}=c_{6}=0, c_{1}+2 c_{2}+c_{3}=\alpha_{123} \epsilon$

$$
\left(c_{1}+c_{2}\right) \frac{\mathrm{d}}{\mathrm{d} t}\left(v_{n} v_{n+1}\right)=\alpha_{123} v_{n} v_{n+1} .
$$

In the first case, the obtained equation is nonlocal. In the second case we have an ODE for $v_{n} v_{n+1}$. Then, as for the lpKdV equation, we pass to a rhombic lattice.

Under the change of variables $k=n+m+1$ and $w_{k+i+j, m+j}=u_{n+i, m+j}$, equation (4.10) becomes

$$
\begin{aligned}
c_{1} w_{k-1, m} w_{k, m} & +c_{2}\left(w_{k, m} w_{k, m+1}+w_{k-1, m} w_{k+1, m+1}\right)+c_{3} w_{k, m+1} w_{k+1, m+1} \\
& +c_{5} w_{k-1, m} w_{k, m+1}+c_{6} w_{k, m} w_{k+1, m+1}=0 .
\end{aligned}
$$

Letting

we transform equation (4.14) into

$$
t=n \epsilon, \quad w_{k, n}=U_{k}(t),
$$

$$
\begin{aligned}
& \left(c_{1}+c_{5}\right) U_{k-1} U_{k}+\left(c_{3}+c_{6}\right) U_{k} U_{k+1}+c_{2}\left(U_{k-1} U_{k+1}+U_{k}^{2}\right) \\
& \quad+\epsilon\left(\left(c_{2} U_{k-1}+\left(c_{3}+c_{6}\right) U_{k}\right) \dot{U}_{k+1}+\left(c_{5} U_{k-1}+c_{2} U_{k}+c_{3} U_{k+1}\right) \dot{U}_{k}\right)+\mathcal{O}\left(\epsilon^{2}\right)=0 .
\end{aligned}
$$

To get a $\mathrm{D} \Delta \mathrm{E}$, at the lowest order in $\epsilon$ we need to set

$$
c_{1}+c_{5}=\epsilon \alpha, \quad c_{3}+c_{6}=\epsilon \beta, \quad c_{2}=\epsilon \gamma,
$$

and choose $c_{1}$ and $c_{6}$ of order one. In this way, at the lowest order in $\epsilon$ we get

$$
\left(c_{6} U_{k+1}+c_{1} U_{k-1}\right) \dot{U}_{k}-\left(\beta U_{k+1}+\gamma U_{k}+\alpha U_{k-1}\right) U_{k}-\gamma U_{k-1} U_{k+1}=0 .
$$

Equation 4.18 satisfies Yamilov S-integrability theorem. Thus, for all values of $c_{i}$, equation (4.10) is an S-integrable and the result obtained by the algebraic entropy is shown to be correct.

4.3. H2 equation: a more complicate equation of the ABS classification. The H2 equation

$$
\begin{aligned}
\left(u_{n, m}\right. & \left.-u_{n+1, m+1}\right)\left(u_{n+1, m}-u_{n, m+1}\right) \\
& +(\beta-\alpha)\left(u_{n, m}+u_{n+1, m}+u_{n, m+1}+u_{n+1, m+1}\right)-\alpha^{2}+\beta^{2}=0,
\end{aligned}
$$

is another of the discrete integrable equations in the Adler-Bobenko-Suris list, see [2] and [11.

As in other cases, we admit that the constants $\alpha$ and $\beta$ depend on the parameter $\epsilon$, in which we carry out the limiting process. We follow the approach, which uses as starting point a background solution provided by equation (4.7a) in [8].

Following [8], we redefine the parameters of H2 4.19):

$$
p=r-a^{2}, \quad q=r-b^{2},
$$

and get the equation:

$$
\begin{aligned}
\left(u_{n, m}\right. & \left.-u_{n+1, m+1}\right)\left(u_{n+1, m}-u_{n, m+1}\right) \\
& +\left(a^{2}-b^{2}\right)\left(u_{n, m}+u_{n+1, m}+u_{n, m+1}+u_{n+1, m+1}+2 r-a^{2}-b^{2}\right)=0 .
\end{aligned}
$$

Among others, equation 4.20 has the solution

$$
u_{0}=(a n+b m+\gamma)^{2}-\frac{1}{2} r .
$$

Making the change 4.2 in 4.21 , we can rewrite 4.20 ) as:

$$
\begin{aligned}
\left(v_{n, m}\right. & \left.-v_{n+1, m+1}\right)\left(v_{n+1, m}-v_{n, m+1}\right) \\
& +2(a-b)\left((a(n+1)+b(m+1)+\gamma) v_{n, m}-(a n+b m+\gamma) v_{n+1, m+1}\right) \\
& -2(a+b)\left((a n+b(m+1)+\gamma) v_{n+1, m}-(a(n+1)+b m+\gamma) v_{n, m+1}\right)=0,
\end{aligned}
$$

which has the solution $v_{0}=0$. 
In order to take the skew limit in one of the indices, as in the previous section we define $k=n+m$ and a new field $w_{k, m}$ according to 4.7$)$. Then 4.22 is transformed into

$$
\begin{aligned}
\left(w_{k, m}\right. & \left.-w_{k+2, m+1}\right)\left(w_{k+1, m}-v_{k+1, m+1}\right)+2(a-b)\left((a(k-m+1)+b(m+1)+\gamma) w_{k, m}\right. \\
& \left.-(a(k-m)+b m+\gamma) w_{k+2, m+1}\right)-2(a+b)\left((a(k-m)+b(m+1)+\gamma) w_{k+1, m}\right. \\
& \left.-(a(k-m+1)+b m+\gamma) w_{k+1, m+1}\right)=0 .
\end{aligned}
$$

In order to take the continuous limit in the index $m$, we replace $a$ and $b$ by $\epsilon \tilde{a}$ and $\epsilon \tilde{b}$ and we define

$$
w_{k+i, m}=U_{k+i}(t), \quad w_{k+i, m+j} \rightarrow U_{k+i}(t)+\epsilon j \dot{U}_{k+i}(t)+O\left(\epsilon^{2}\right) .
$$

Then, the lower order terms of (4.23) are

$$
\begin{aligned}
\left(U_{k}\right. & \left.-U_{k+2}-\epsilon \dot{U}_{k+2}\right)\left(U_{k+1}-U_{k+1}-\epsilon \dot{U}_{k+1}\right) \\
& +2 \epsilon(\tilde{a}-\tilde{b})\left((\epsilon \tilde{a}(k-m+1)+\epsilon \tilde{b}(m+1)+\gamma) U_{k}\right. \\
& \left.-(\epsilon \tilde{a}(k-m)+\epsilon \tilde{b} m+\gamma)\left(U_{k+2}+\epsilon \dot{U}_{k+2}\right)\right) \\
& -2 \epsilon(\tilde{a}+\tilde{b})\left((\epsilon \tilde{a}(k-m)+\epsilon \tilde{b}(m+1)+\gamma) U_{k+1}\right. \\
& \left.\left.-(\epsilon \tilde{a}(k-m+1)+\epsilon \tilde{b} m+\gamma) U_{k+1}+\epsilon \dot{U}_{k+1}\right)\right)+\cdots=0,
\end{aligned}
$$

which has no order zero term. The first order term of (4.24) is

$$
\dot{U}_{k}=2 \gamma(\tilde{a}-\tilde{b})\left(U_{k-1}-U_{k+1}\right),
$$

and this is an equation satisfying Yamilov's theorem.

4.4. Equation ${ }_{r} H_{1}^{\epsilon}$ from ABS extended classification. We consider the following equation:

$$
\begin{aligned}
{ }_{r} H_{1}^{\epsilon}= & (\alpha-\beta)\left(\epsilon^{2} \chi_{m+n} u_{n+1, m} u_{n, m+1}+\epsilon^{2} \chi_{m+n+1} u_{n+1, m+1} u_{n, m}-1\right) \\
& +\left(u_{n, m}-u_{n+1, m+1}\right)\left(u_{n+1, m}-u_{n, m+1}\right)=0,
\end{aligned}
$$

where $\alpha, \beta, \epsilon$ are three parameters, which could depend on the steps of the lattice and

$$
\chi_{m}=\frac{1}{2}\left(1+(-1)^{m}\right) \text {. }
$$

Equation (4.26) is an $S$-integrable equation of Boll classification [4 appearing in the ABS list [3, [1].

As in the previous case, we transform the variable $u_{n, m} \rightarrow v_{n, m}$ in such a way that the resulting equation has $v_{0}=0$ as a particular solution:

$$
u_{n, m}=v_{n, m}+f,
$$

where $f$, a constant, must satisfy the identity

$$
(\alpha-\beta)\left(\epsilon^{2} f^{2}-1\right)=0 .
$$

Choosing one of the two signs for $f$ in 4.28 with $\epsilon \neq 0$, we get:

$$
u_{n, m}=v_{n, m}+\frac{1}{\epsilon} \text {. }
$$

The equation for $v_{n, m}$ is

$$
\begin{aligned}
(\alpha-\beta)\left(\chi_{n+m+1}\left(1+\epsilon v_{n, m}\right)\left(1+\epsilon v_{n+1, m+1}\right)\right. & \left.+\chi_{n+m}\left(1+\epsilon v_{n, m+1}\right)\left(1+\epsilon v_{n+1, m}\right)-1\right) \\
& +\left(v_{n+1, m}-v_{n, m+1}\right)\left(v_{n, m}-v_{n+1, m+1}\right)=0
\end{aligned}
$$

and $v_{0}=0$ is a solution of 4.30 .

In order to take a semi-continuous skew limit, we first modify the lattice from a rectangular one to an oblique lattice by defining a new index $k=n+m$ and leaving $m$ as it is. Then we 
take the continuous limit in the $m$ direction of the new lattice. The equation with $w_{k, m}$ defined as in (4.7) now reads as

$$
\begin{aligned}
(\alpha-\beta)\left(\chi_{k+1}\left(1+\epsilon w_{k, m}\right)\left(1+\epsilon w_{k+2, m+1}\right)\right. & \left.+\chi_{k}\left(1+\epsilon w_{k+1, m+1}\right)\left(1+\epsilon w_{k+1, m}\right)-1\right) \\
& +\left(w_{k+1, m}-w_{k+1, m+1}\right)\left(w_{k, m}-w_{k+2, m+1}\right)=0 .
\end{aligned}
$$

Taking the continuous limit in the $m$ direction and assuming that the order parameter is $\epsilon$, we find that

$$
w_{k+i, m}=U_{k+i}, \quad w_{k+i, m+1}=U_{k+i}+\epsilon \dot{U}_{k+i}+O\left(\epsilon^{2}\right),
$$

and substituting 4.32 in 4.31, we get

$$
\begin{aligned}
& (\alpha-\beta)\left(\chi_{k+1}\left(1+\epsilon U_{k}\right)\left(1+\epsilon\left(U_{k+2}+\epsilon \dot{U}_{k+2}+O\left(\epsilon^{2}\right)\right)\right)\right. \\
& \left.\quad+\chi_{k}\left(1+\epsilon\left(U_{k+1}+\epsilon \dot{U}_{k+1}+O\left(\epsilon^{2}\right)\right)\right)\left(1+\epsilon U_{k+1}\right)-1\right) \\
& \quad+\left(U_{k+1}-U_{k+1}-\epsilon \dot{U}_{k+1} O\left(\epsilon^{2}\right)\right)\left(U_{k}-U_{k+2}-\epsilon \dot{U}_{k+2}+O\left(\epsilon^{2}\right)\right)=0 .
\end{aligned}
$$

Taking into consideration the definition 4.27) of $\chi_{m}$, we see that the zero order terms in (4.33) vanish. The first order terms are

$$
(\alpha-\beta)\left(\chi_{k+1}\left(U_{k}+U_{k+2}\right)+2 \chi_{k} U_{k+1}\right)-\left(U_{k}-U_{k+2}\right) \dot{U}_{k+1}=0 .
$$

Equation (4.34) is a D $\Delta \mathrm{E}$ satisfying an extension of Yamilov's theorem when the function $f$ appearing in (2.1) depends explicitly on the index $n$ :

$$
\dot{U}_{k}=(\alpha-\beta) \frac{2 \chi_{k-1} U_{k}+\chi_{k}\left(U_{k-1}+U_{k+1}\right)}{U_{k-1}-U_{k+1}} .
$$

Equation 4.35 is a subcase of the $k$-dependent generalization of the Yamilov discretization of the Krichever-Novikov equation postulated in [9] and discussed in [11].

\section{Conclusions}

In this paper we have discussed Yamilov's theorem on the form of evolutionary D $\Delta$ Es from the point of view of its consequences for PDEs and P $\Delta$ Es. In particular, we have shown that this theorem have no implication for the S-integrability of PDEs.

We have not been able to have such complete result in the case of P $\Delta$ Es. In all considered cases, the theorem is satisfied, even in the case of $\mathrm{P} \Delta \mathrm{Es}$ with non-constant coefficients.

Often, unless we use the skew limit, we obtain nonlocal evolutionary P $\Delta$ Es. Some natural questions are

- Is there an extension of Theorem 2.1 when the function $f$ depends explictly on $n$ ?

- Is (4.6) also S-integrable?

- Is there an extension of Theorem 2.1 which shows the S-integrability of (4.6)?

- Has Theorem 2.1 implications for the construction of numerical schemes for S-integrables PDEs?

We plan to continue this research by answering these questions and looking for a compulsory shape in the case of S-integrable P $\Delta$ Es.

\section{REFERENCES}

1. M. Ablowitz, J. Ladik. Nonlinear differential-difference equations // J. Math. Phys. 16:3, 598-603 (1975).

2. V.E. Adler, A.I. Bobenko, Yu.B. Suris. Classification of Integrable Equations on Quad-Graphs. The Consistency Approach // Comm. Math. Phys. 233:3, 513-543 (2003).

3. V.E. Adler, A.I. Bobenko, Yu.B. Suris. Discrete nonlinear hyperbolic equations: classification of integrable cases // Funct. Anal. Appl. 43:1, 3-17 (2009). [arXiv:0705.1663]. 
4. R. Boll. Classification of $3 D$ consistent quad-equations // J. Nonlin. Math. Phys. 18:3, 337-365 (2011).

5. F. Calogero. Why are certain nonlinear PDEs both widely applicable and integrable // in What is integrability?, ed. by V. E. Zakharov, Springer-Verlag, Berlin, 1-61 (1991).

6. J. Hietarinta, N. Joshi, F.W. Nijhoff. Discrete systems and integrability, Cambridge University Press, Cambridge (2016).

7. J. Hietarinta, C. Viallet. Searching for integrable lattice maps using factorization // J. Phys. A: Math. Theor. 40:42, 12629-12643 (2007).

8. J. Hietarinta, D. Zhang. Soliton solutions for ABS lattice equations: II. Casoratians and bilinearization // J. Phys. A: Math. Theor. 42:40, 404006 (2009).

9. D. Levi, R. Yamilov. Conditions for the existence of highers symmetries of evolutionary equations on the lattice // J. Math. Phys. 38:12, 6648-6674 (1997).

10. D. Levi, R.I. Yamilov. Generalized Lie symmetries for difference equations // in Symmetries and integrability of difference equations, ed. by D. Levi, P. Olver, Z. Thomova, P. Winternitz, Cambridge University Press, Cambridge, 160-190 (2011).

11. D. Levi, P. Winternitz, R.I. Yamilov. Continuous Symmetries and Integrability of Discrete Equations, Amer. Math. Soc. and the Centre de Recherches Mathématiques, to be published (2021).

12. F. Nijhoff, H. Capel. The discrete Korteweg-de Vries equation // Acta Appl. Math. 39, 133-158 (1995).

13. R. Yamilov. Symmetries as integrability criteria for differential difference equations // J. Phys. A: Math. Gen. 39:45, R541-R623 (2006).

Decio Levi,

Mathematical and Physical Department

Roma Tre University

Via della Vasca Navale, 84,

I00146 Roma, Italy

E-mail: deciolevi_work@yahoo.com

Miguel A. Rodríguez,

Dept. Física Teórica

Universidad Complutense de Madrid

Pza. de las Ciencias, 1,

28040 Madrid, Spain

E-mail: rodrigue@ucm.es 